\title{
An Optimization Method of the Maximum Torque per Ampere for the Self-tuning of the PMSM Robust
}

\author{
Chi Jie-Fu, Hu Yan-Kui and Zhao Kai \\ School of Automation and Electrical Engineering, Lanzhou Jiaotong University, \\ Lanzhou, China \\ Teaching Affairs Division, Pingdingshan University, Henan Pingdingshan, \\ 467000, China \\ 1227504734@qq.com
}

\begin{abstract}
For the parameter dependency problem exists in the efficiency optimization on the speed control system of the traditional permanent magnet synchronous motor (hereinafter referred to as the PMSM), an optimization method of the maximum torque per ampere (hereinafter referred to as the MTPA) for the self-tuning of the PMSM robust is proposed. By overlapping a small high frequency sinusoidal signal on the vector angle of the stator current, the digital signal processing is adopted to extract the power signal to reflect the change rule of the torque, and the PI regulator is locked for the best vector angle of the stator current. In addition, the inner current regulator compensates the dynamic response performance of the robust MTPA. Finally, the dynamic and steady-state performance and operating efficiency of the robust MTPA proposed is verified by the $22 \mathrm{kw}$ experimental prototype.
\end{abstract}

Keywords: high-voltage inverter, capacitor voltage balance, high-frequency signal injection, permanent magnet synchronous motor

\section{Introduction}

In the driving system of the variable frequency air condition compressor, the PMSM gets an extremely wide range of industrial applications ${ }^{[1-3]}$ with its unique high efficiency, high power factor and high power density. As a well-known PMSM magnetic material, the advantage of high magnetic density of the rare earth neodymium iron boron (hereinafter referred to as the Nd-Fe-B) makes it of great significance ${ }^{[4-5]}$ in the practical application in the compressor with demanding requirements of the motor's volume. However, in consideration of the long-time working of the air condition compressor system under high temperature, it will easily cause the overheating phenomenon in the PMSM. In view of the significant change of the field density of Nd-Fe-B with the change of the environment temperature, it makes the high volatility in the rotor flux under the environment of high temperature, and causes the deterioration ${ }^{[6-8]}$ in the performance of the PMSM output torque under the same current situation ${ }^{[9,10]}$.

This paper proposes an optimization method of the self-tuning MTPA of the robust for the parameter dependency problem exists in the MTPA control for the speed control system of the traditional PMSM. By overlapping a small high frequency sinusoidal signal on the vector angle of the stator current, the digital signal processing is adopted to extract the power signal to reflect the change rule of the torque, and the PI regulator is locked for the best vector angle of the stator current. In addition, the inner current regulator compensates the dynamic response performance of the robust MTPA. Finally, the dynamic and steady-state performance of the robust MTPA proposed is experimentally verified by the experimental prototype of a $22 \mathrm{kw}$ PMSM HEV system and the result 
reveals that the HEV system is equipped with strong robustness and high efficiency in this method.

\section{Systematic Mathematical Modeling}

\subsection{Pmsm Mathematical Model}

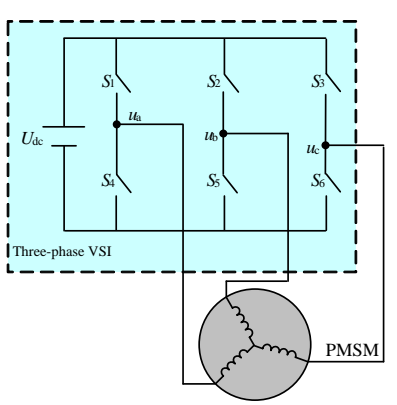

(a) PMSM system structure drawing

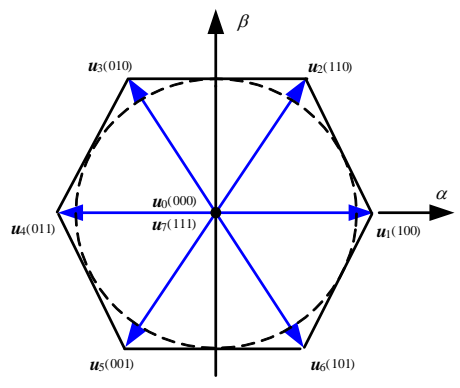

(b) Voltage space vector diagram

Figure 1. Pmsm Speed Control System Overview

PMSM speed control system overview is shown in Figure 1, for the three-phrase ideal symmetric PMSM system, the influence of the stator resistance is neglected and the following electromagnetic torque equation under the two-phase rotating $d q$ coordinate system is established:

$$
T_{\mathrm{e}}=\frac{3}{2} n_{\mathrm{p}}\left[\psi_{\mathrm{m}} i_{\mathrm{q}}-\left(L_{\mathrm{q}}-L_{\mathrm{d}}\right) i_{\mathrm{d}} i_{\mathrm{q}}\right]
$$

In the formula: $T_{\mathrm{e}}$ is the electromagnetic torque of the motor; $n_{\mathrm{p}}$ is the pole-pairs of the motor; $\Psi_{\mathrm{m}}$ is the flux linkage of the permanent magnet; $i_{\mathrm{d}}$ and $i_{\mathrm{q}}$ are the stator current on the axle $d q ; L_{\mathrm{d}}$ and $L_{\mathrm{q}}$ are the inductance on the axle $d q$.

Set that vector of the current vector $\boldsymbol{i}_{\mathrm{dq}}$ and $q$ axis is in the $\beta$ angle, by this time the current component under the coordinate system of $d q$ is

$$
\begin{aligned}
& i_{\mathrm{d}}=-\left|\boldsymbol{i}_{\mathrm{dq}}\right| \sin (\beta) \\
& i_{\mathrm{q}}=\left|\boldsymbol{i}_{\mathrm{dq}}\right| \cos (\beta) \\
& \left|\boldsymbol{i}_{\mathrm{dq}}\right|=i_{\mathrm{m}}=\sqrt{i_{\mathrm{d}}^{2}+i_{\mathrm{q}}^{2}}
\end{aligned}
$$

In the formula: $i d q$ is the space vector on the axle dq; im is the amplitude of the stator current. Enter the formula (2) into the torque equation of the formula (1) and get

$$
\begin{aligned}
T_{\mathrm{e}}= & \frac{3}{2} n_{\mathrm{p}}\left[\psi_{\mathrm{m}} i_{\mathrm{m}} \cos (\beta)+\right. \\
& \left.\left(L_{\mathrm{q}}-L_{\mathrm{d}}\right) i_{\mathrm{m}}^{2} \sin (\beta) \cos (\beta)\right]
\end{aligned}
$$

\subsection{Mtpa Optimal Trajectory}

Assume that the amplitude $i_{\mathrm{m}}$ of the stator current in the formula (3) is a fixed value, and we can see that the electromagnetic torque of the motor is only related with the included angle $\beta$. In order to confirm the extreme point of the formula (5), the differential operation with $\beta$ as the variable is 


$$
\begin{aligned}
\frac{d T_{\mathrm{e}}}{d \beta} & =\frac{3}{2} n_{\mathrm{p}}\left[-\psi_{\mathrm{m}} i_{\mathrm{m}} \sin (\beta)+\left(L_{\mathrm{q}}-L_{\mathrm{d}}\right) i_{\mathrm{m}}^{2} \cos (2 \beta)\right] \\
& =0
\end{aligned}
$$

Change the short form (6) and get

$$
\beta=\arccos \left[\frac{-\psi_{\mathrm{m}}+\sqrt{\psi_{\mathrm{m}}^{2}+8 i_{\mathrm{m}}^{2}\left(L_{\mathrm{q}}-L_{\mathrm{d}}\right)^{2}}}{4\left(L_{\mathrm{q}}-L_{\mathrm{d}}\right) i_{\mathrm{m}}}\right]
$$

Neglect the influence of the stator resistance drop of the PMSM, and by this time the current and amplitude limits are

$$
\begin{gathered}
i_{\mathrm{d}}^{2}+i_{\mathrm{q}}^{2} \leq I_{\text {max }}^{2} \\
L_{\mathrm{d}}^{2}\left(i_{\mathrm{d}}+\frac{\psi_{\mathrm{m}}}{L_{\mathrm{d}}}\right)^{2}+L_{q}^{2} i_{q}^{2} \leq \frac{V_{s}^{2}}{\omega_{e}^{2}}
\end{gathered}
$$

We can see that the key to realize MTPA is how to reasonably allocate the proportion of the current $i_{\mathrm{d}}$ and $i_{\mathrm{q}}$ to realize the maximum electromagnetic torque output of PMSM. The function of the torque angle $\beta$ in ideal conditions is given in formula (5). It is an advanced and complex function composed by the $L_{\mathrm{d}}$ and $L_{\mathrm{q}}$ as well as the rotor flux $\psi_{\mathrm{m}}$. The torque angles get by the second order polynomial fitting method are mostly adopted in the practical engineering application.

\section{Operating Principle of the MTPA for Robust's Self-Tuning}

In consideration of the rapid change in aspects of load and temperature occurred during the running process of the HEV system, great changes appear in different operating positions for the system parameters $L_{\mathrm{d}}, L_{\mathrm{q}}$ and $\psi_{\mathrm{m}}$, and it makes the operating position deviate from the position of MTPA.

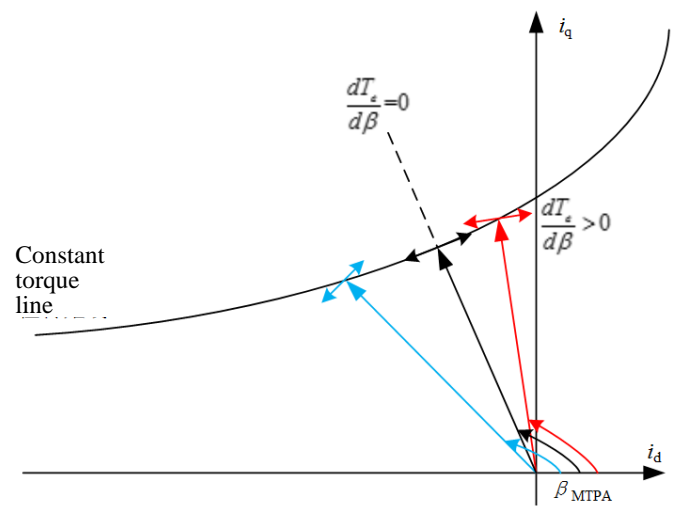

Figure 2. Variation of Current Vector Angle $B$ and Torque Te

The variation of current vector angle $\beta$ and torque Te is shown in Figure 2, thereinto the $\beta_{\mathrm{MTPA}}$ is the curve tangent point of the constant torque, and by this time $d T_{\mathrm{e}} / d \beta=0$; in case $\beta>\beta_{\mathrm{MTPA}}$, then $d T_{\mathrm{e}} / d \beta<0$; in contrary, in case $\beta<\beta_{\mathrm{MTP}}$, then $d T_{\mathrm{e}} / d \beta>0$. Thus whether the motor is operating on the optimal point of MTPA can be confirmed through detecting that whether the value of the $d T_{\mathrm{e}} / d \beta$ is zero, without relying on the complex PMSM mathematical model with sensitive parameters.

For the torque value which is hard to measure or calculate in the practical application, it proposes a method to get the $\mathrm{dTe} / \mathrm{d} \beta$ based on the high frequency auxiliary signal injection method in this paper, so as to realize the optimal MTPA trajectory tracking for 
the PMSM. By this time, a high frequency small signal is injected into the current reference vector angle $\beta$, namely,

$$
\beta=\beta_{\text {avg }}+\beta_{\mathrm{h}}=\beta_{\text {avg }}+A_{\text {mag }} \sin \left(f_{\mathrm{h}} \times 2 \pi t\right)
$$

In the formula: $\beta_{\text {avg }}$ is the average value of the voltage vector angle; $\beta_{\mathrm{h}}$ is the vector angle of the high frequency fluctuation; $A_{\operatorname{mag}}$ and $f_{\mathrm{h}}$ are the amplitude and frequency of the vector angle of the high frequency fluctuation, thereinto the $A_{\text {mag }}$ must be small enough (compared with the practical running current of PMSM) for the purpose that the influence on the speed control may be neglected and the $f_{\mathrm{h}}$ must be bigger than the width of the speed loop and far smaller than the switching frequency $(5 \mathrm{kHz})$ of the inverter. Thus the high-frequency signal $A_{\text {mag }}=0.05 \mathrm{~A}$ and $f_{\mathrm{h}}=300 \mathrm{~Hz}$ are injected.

Due to the function of the high-frequency injected signal, corresponding changes occur on the current, voltage, power and other variables of PMSM, thereinto the relationship between the mechanical power of the motor $P_{\text {mech }}$ and the torque $T_{\mathrm{e}}$ is as follow

$$
T_{\mathrm{e}}(\beta)=\frac{P_{\mathrm{mech}}(\beta)}{\omega_{\mathrm{r}}}
$$

In the formula: $T_{e}(\beta)$ and $P_{\text {mech }}(\beta)$ are the corresponding electromagnetic torque and mechanical power of the current vector angle $\beta ; \omega_{\mathrm{r}}$ is the rotor angular velocity.

In order to understand the idea of the high-frequency injection, the Taylor series decomposition is made on the torque equation and it gets

$$
T_{\mathrm{e}}(\beta+\Delta \beta)=T_{\mathrm{e}}(\beta)+\frac{\partial T_{\mathrm{e}}}{\partial \beta} \Delta \beta+\frac{\partial}{\partial \beta}\left(\frac{\partial T_{\mathrm{e}}}{\partial \beta}\right) \Delta \beta^{2} \mathrm{~L}
$$

In the formula: $\Delta \beta$ is the fluctuation value of the current vector angle; $\partial T_{\mathrm{e}} / \partial$ is the partial differential calculation of the torque.

In consideration of the small amplitude of the high-frequency signal injected and that the frequency is large enough, the control and regulation of the speed loop will not be affected, and the items with second order or above in formula (10) may be neglected due to their small values. The electromagnetic power can be converted into the equation as follow where the torque is changed as the current vector angle changes

$$
\left\{P_{\mathrm{e}}\right\} \propto\left\{T_{\mathrm{e}}(\beta)+\frac{\partial T_{\mathrm{e}}}{\partial \beta} A \sin \omega_{h} t\right\} \propto \frac{\partial T_{\mathrm{e}}}{\partial \beta}
$$

In the formula: $P_{\mathrm{e}}$ is the electromagnetic input power of the motor; $\omega_{\mathrm{h}}$ is the angular frequency high frequency signal.

We can see from the abovementioned formula that if the high frequency auxiliary signal injection method is adopted, the MTPA of PMSM could be realized. Thus, for the power after injecting the high-frequency signal, after a series of signal processing, the response of the mechanical power on the signal injected is extracted, and this response signal just could reflect the changes of the value of $d T_{\mathrm{e}} / d \beta$. Then the controller is applied to maintain the $d T_{\mathrm{e}} / d \beta$ as zero so as to lock the optimal stator vector angel $\beta$, and realize the MTPA control of the PMSM.

\section{High-Frequency Control System Design}

It is the PMSM based on high-frequency signal injection MTPA overall control block diagram in Figure 3. It adopts the basic method of high frequency auxiliary signal (8) injection to overlap a small high-frequency signal component on the stator current vector angle for testing the mechanical power response caused by the high-frequency signal injected through digital signal processing, and acquiring the set value of the current vector angle to realize the operation of MTPA through designing an automatic regulation 
mechanism. In addition, one high-frequency current signal control loop is increased and different control methods are applied for the fundamental wave signal and high-frequency signal. In this chapter, the high-frequency power signal processing and the high-frequency current controller design will be discussed in detail.

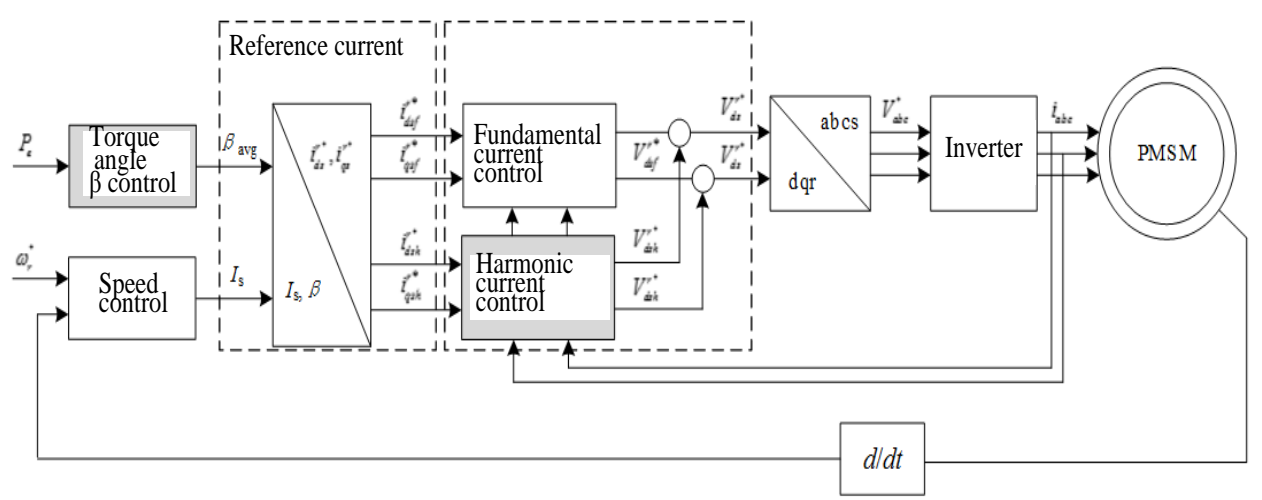

\section{Figure 3. Overall Control Diagram of the MTPA for Air Condition Compressor Based on High-Frequency Signal Injection}

\subsection{High-Frequency Power Signal Processing}

Due to the high frequency auxiliary signal shown in the injection formula (8), when assuming that the is far smaller than, the quadrature and direct axis current of PMSM could be separately expressed as

$$
\begin{aligned}
i_{\mathrm{d}} & =I_{\mathrm{s}} \cos \left(\beta_{\text {avg }}+A_{\mathrm{mag}} \sin \omega_{\mathrm{h}} t\right) \\
& \approx I_{\mathrm{s}} \cos \beta_{\text {avg }}-I_{\mathrm{s}} A_{\text {mag }} \sin \beta_{\text {avg }} \sin \omega_{\mathrm{h}} t=i_{d f}+i_{d h} \\
i_{\mathrm{q}}= & I_{\mathrm{s}} \sin \left(\beta_{\text {avg }}+A_{\mathrm{mag}} \sin \omega_{\mathrm{h}} t\right) \\
& \approx I_{\mathrm{s}} \sin \beta_{\text {avg }}-I_{\mathrm{s}} A_{\text {mag }} \cos \beta_{\text {avg }} \sin \omega_{\mathrm{h}} t=i_{q f}+i_{q h}
\end{aligned}
$$

In the formula: $I_{\mathrm{s}}$ is the current vector amplitude; $\underline{i}_{\mathrm{df}}$ and $i_{\mathrm{qf}}$ are the high-frequency current component on the axle $d q$.

The electromagnetic input power of the motor includes copper loss, active power and mechanical power, in consideration of the high-frequency signal injected, they can be expressed respectively as

$$
P_{\mathrm{e}}=P_{\text {copper }}+P_{\text {reactive }}+P_{\text {mech }}=\frac{3}{2}\left(v_{d} i_{d}+v_{q} i_{q}\right)
$$

In the formula: $P_{\text {cooper }}$ is the copper loss of the motor; $P_{\text {reactive }}$ is the active power; $P_{\text {mech }}$ is the mechanical power.

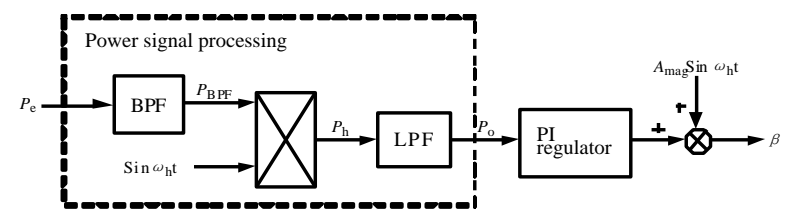

Figure 4. Principle of Signal Frequency Extracting Method

As shown in Figure 4, in order to extract the component of the signal frequency injected from the tested power, firstly the direct component and harmonic component 
shall be filtered from the electromagnetic power signal through a band-pass filter with $f_{\mathrm{h}}$ as the center frequency. The signal obtained is multiplied with the last unit sinusoidal signal with the same frequency of the signal injected, finally it gets through a low pass filter which is much less than $f_{\mathrm{h}}$ in the cut-off frequency, and get the direct component $P_{\mathrm{o}}$ which is directly proportional to $d T_{\mathrm{e}} / d \beta$ after filtering the high-frequency component

$$
\begin{aligned}
P_{\mathrm{o}} & =\frac{3}{4} \omega_{r} A_{\text {mag }} I_{s}^{2}\left[\left(L_{d s}-L_{q s}\right) I_{s} \cos 2 \beta_{\text {avg }}+\psi_{\mathrm{m}} \cos \beta_{\text {avg }}\right] \\
& \propto \frac{d T_{e}}{d \beta}
\end{aligned}
$$

By this time, the result $P_{\mathrm{o}}$ of the power signal processing procedure is controlled as zero by introducing the PI regulator to ensure that the $d T_{\mathrm{e}} / d \beta$ is zero, namely to find the tangent point of the power curve, lock the optimal current vector angle $\beta_{\mathrm{opt}}$, and the motor is operating under the state of MTPA is guaranteed accordingly.

\subsection{Design ot the High Frequency Current Controller}

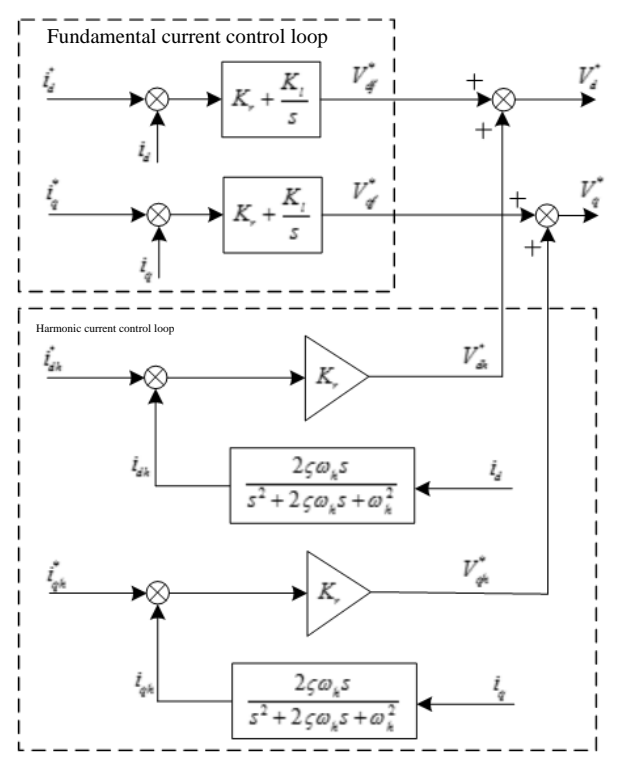

Figure 5. High Frequency Current Signal Control Loop

As the MTPA control method adopted is to monitor the input power's response to the injected signal frequency, the change of the power shall only be influenced and generated by the injected signal. However, in practice, the power may be changed due to the influence of many factors, such as changed to variable speed operation, torque disturbance and varying load. In order to extract the power and the variation of the signal injected from other changes, the frequency of the signal injected shall be as high as possible. Generally speaking, it will reach a few hundred hertz. For this point, a special high-frequency current signal control loop is designed in this paper to improve the response of the current inner loop, and its control is shown in Figure 5.

We can see from Figure 5 that for the fundamental frequency current loop, it applies the common PI control, while for the high-frequency current loop, firstly the components of the injected signal frequency shall be extracted from the feedback current. Namely, the ratio control is made for the difference value through a second order bandpass filter with an value ratio of $K_{p}$, and the transfer function of the system could be expressed as 


$$
\frac{i}{i_{h}^{*}}=\frac{K_{p} s^{2}+2 \zeta \omega K_{p} s+\omega^{2} K_{p}}{L s^{3}+(2 \zeta \omega L+R) s^{2}+\left[\omega^{2} L+2 \zeta \omega(K p+R)\right] s+\omega^{2} R}
$$

In the formula: $L$ and $R$ are the stator and other equivalent resistance of PMSM; $\zeta$ is the damping ratio.

The Bode plots for the High-frequency current control loop are shown in Figure 6. It can be seen from Figure 6 that in the target frequency of $500 \mathrm{~Hz}$, the system's gain is $0 \mathrm{~dB}$ and the phase shift is 0 degree. Thereinto the impact of the value of $\zeta$ is small. According to the principle of automatic control, the smaller the value of $\zeta$ is, the steeper the curve in Figure 7 will be, and the $K_{p}$ is generally taken for more than ten times of the high-frequency impedance value.

It can be seen from the capacitor voltage fluctuation rules of the sub-module that the fluctuation frequency and amplitude of the bridge arm power can affect the capacitor voltage fluctuations. In case that the fluctuation amplitude of the bridge arm power is kept unchanged, the fluctuation amplitude of the capacitor voltage could be reduced through promoting the fluctuation frequency of the bridge arm power. By reasonable injection of high-frequency zero sequence voltage, the high-frequency component will occur on the bridge arm current and bridge arm voltage, the bridge arm power fluctuations will transfer from low frequency to high frequency, and it can reduce the fluctuation amplitude of the capacitor voltage in low-frequency operation.

Suppose that the high-frequency zero sequence voltage overlapped for the bridge arm is $v_{\mathrm{z}}$, the high-frequency multi-phase circulation overlapped on phase a is $i_{\text {pza }}$, by this time the current and voltage of the phase a bridge arm are respectively:

$$
\left\{\begin{array}{l}
i_{\mathrm{pa}}^{\prime}=\frac{I_{\mathrm{dc}}}{3}+\frac{I_{\mathrm{a}}}{2}+i_{\mathrm{pza}} \\
i_{\mathrm{n} a}^{\prime}=\frac{I_{\mathrm{dc}}}{3}+\frac{I_{\mathrm{a}}}{2}+i_{\mathrm{pza}} \\
v_{\mathrm{pa}}^{\prime}=-v_{\mathrm{ao}}-v_{\mathrm{z}}+\frac{V_{\mathrm{dc}}}{2} \\
v_{\mathrm{na}}^{\prime}=v_{\mathrm{ao}}+v_{\mathrm{z}}+\frac{V_{\mathrm{dc}}}{2}
\end{array}\right.
$$

By this time, the output instantaneous power on the upper and lower bridge arms of phase are respectively:

$$
\left\{\begin{array}{l}
P_{\mathrm{pa}}^{\prime}=-v_{\mathrm{pa}}^{\prime} i_{\mathrm{pa}}^{\prime}=-P_{1}+P_{2}+P_{3} \\
P_{\mathrm{na}}^{\prime}=-v_{\mathrm{na}}^{\prime} i_{\mathrm{na}}^{\prime}=P_{1}-P_{2}+P_{3}
\end{array}\right.
$$

In the formula: $P_{1}, P_{2}$ and $P_{3}$ are respectively:

$$
\left\{\begin{array}{l}
P_{1}=\frac{V_{\mathrm{dc}} i_{a}\left[1-m^{2} \sin ^{2}(\omega t)\right]}{4}-v_{z} i_{p z a} \\
P_{2}=\frac{V_{\mathrm{m}} i_{\mathrm{a}} v_{\mathrm{z}} \sin (\omega t)}{V d c}+V_{m} i_{p z a} \sin (\omega t) \\
P_{3}=\frac{i_{a} v_{z}-V_{d c} i_{p z a}}{2}
\end{array}\right.
$$

It can be seen from the formula (18) that after the high-frequency components $\mathrm{VZ}$ and ipza are overlapped, P2 and P3 will only contain the high-frequency components, while PI still contains the low-frequency components. If the bridge arm power only contains high-frequency components, the low-frequency components in P1 should be eliminated or make it equal to 0 . Make $\mathrm{P} 1=0$, use the product to sum characteristics of the sine function, the components of the high-frequency components vzipza which are in the equal size and converse symbols with the low-frequency component Vdcia $[1-\mathrm{m} 2 \sin 2$ 
(omega t) / 4] of PI by product to sum operation, and the influence of the high-frequency components could be offset. The multi-phase circulation ipza is:

$$
i_{\mathrm{pza}}=\frac{V_{\mathrm{dc}} i_{\mathrm{a}}\left[1-m^{2} \sin ^{2}(\omega t)\right]}{4 v_{z}}
$$

The expression of the high-frequency zero sequence voltage is set as

$$
v_{\mathrm{z}}=V_{z} \sin \left(\omega^{\prime} t\right)
$$

In the formula: $V_{\mathrm{z}}$ is the zero sequence voltage peak, and $\omega$ is the zero sequence voltage angular frequency.

When applying CPS-SPWM for modulation for MMC, the phase voltage peak and the zero sequence voltage peak shall meet:

$$
V_{\mathrm{m}}+V_{z}=m \frac{V_{\mathrm{dc}}}{2}+V z \leq \frac{V_{\mathrm{dc}}}{2}
$$

It can be seen from formula (22) that the zero sequence voltage peak $V_{\mathrm{Z}}$ shall choose the max. value

$$
V_{\mathrm{z}}=(1-m) \frac{V_{\mathrm{dc}}}{2}
$$
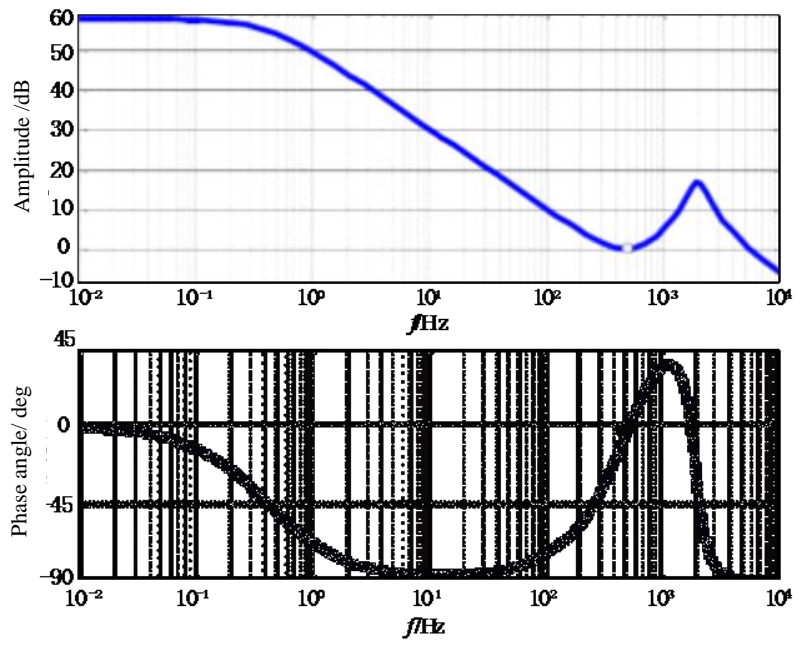

Figure 6. Bode Plots for the High-Frequency Current Control Loop

\section{Experimental Verification}

\subsection{Experimental Platform and Solution}

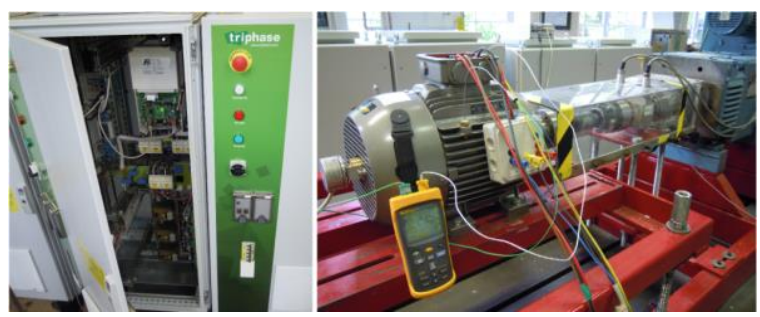

Figure 7. 22kw Prototype of Permanent Magnet Synchronous Motor 
In order to verify the feasibility and effectiveness of robust MTPA method of air condition compressor based on the high-frequency signal injection, the experimental platform of the speed control system for $22 \mathrm{kw}$ PMSM as shown in Figure 7 is established, and the main parameters of the mentioned experiment platform are given in Table 1. The core algorithm in the experiment is the DSP of TI Company in model TMS320F2812, and its major task is to complete the core algorithm of robust MTPA operation, system communication and other functions; Coprocessors are FPGA and CPLD in model Xilinx, there into FPGA is mainly for the functions of AD sampling, data storage and other functions, and CPLD is mainly for the functions of PWM state detection, dead-time compensation, pulse inhibit and other functions. The parameters of the experimental platform and simulation parameters are the same. The variables required for observation in the experiment is observed with the Agilent MSO6054A oscilloscope after the D/A output in the control panel, the sample data is imported into the computer with the sampling frequency of $100 \mathrm{kHz}$ and the Matlab software is applied for the graphical output.

Table 1. Main Parameters of PMSM System Experimental Platform

\begin{tabular}{|c|c|}
\hline Rated voltage/V & 380 \\
\hline Rated current $/ \mathrm{A}$ & 44 \\
\hline Stator resistance/ $\boldsymbol{\Omega}$ & 0.86 \\
\hline Reactance on $d$ axle $/ \mathrm{mH}$ & 4.5 \\
\hline Reactance on $q$ axle $/ \mathrm{mH}$ & 31.7 \\
\hline Rotor flux $/ \mathrm{Wb}$ & 1.2 \\
\hline Pole-pairs & 3 \\
\hline DC busbar voltage $/ \mathrm{V}$ & 600 \\
\hline
\end{tabular}

\subsection{Experiment Results and Analysis}

The robust self-tuning MTPA general control features is shown in Figure 8 and the motor is controlled with the rated speed of $600 \mathrm{r} / \mathrm{min}$. During the stage of $t=[0 \sim 0.05 \mathrm{~s}]$, the PMSM is accelerated from $0 \mathrm{r} / \mathrm{min}$ to $600 \mathrm{r} / \mathrm{min}$. During this stage, in order to get a max. torque output, the current amplitude $i_{\mathrm{m}}$ reaches the allowed max. current amplitude, namely $i_{\mathrm{m}}=i_{\max }=40 \mathrm{~A}$; during the stage of $t=[0.05 \sim 0.1 \mathrm{~s}]$, the PMSM is under the positive load operation, by this time the MTPA regulator is injected with high frequency to rapidly complete the closed-loop tracking, lock the optimal current vector angle $\beta$, and complete the reasonable distribution of $i_{\mathrm{d}}=-15 \mathrm{~A}$ and $i_{\mathrm{q}}=32 \mathrm{~A}$ for the axles $d$ and $q$. The time consumed is about $5 \mathrm{~ms}$ during the dynamic tuning procedure for the whole MTPA, and the rapid response (about 2ms) of the PMSM electromagnetic torque $T_{\mathrm{e}}$ is ensured. During the stage of $t=[0.1 \sim 0.2 \mathrm{~s}]$, PMSM gradually enters into the reverse loading stage. With the change of the load torque, the self-adaptive tuning could be effectively available for the exciting current $i_{\mathrm{d}}$, and PMSM is operating on the optimal track of MTPA all the time during this procedure.

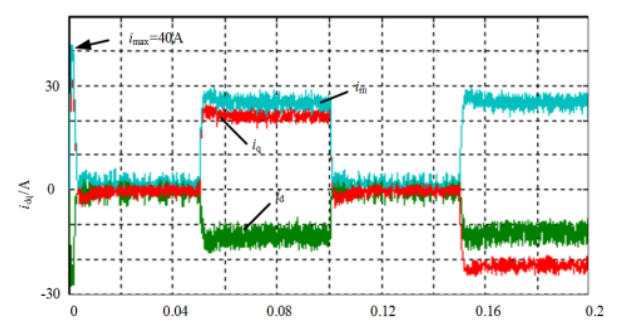

(A) Stator Current on Dq Axle

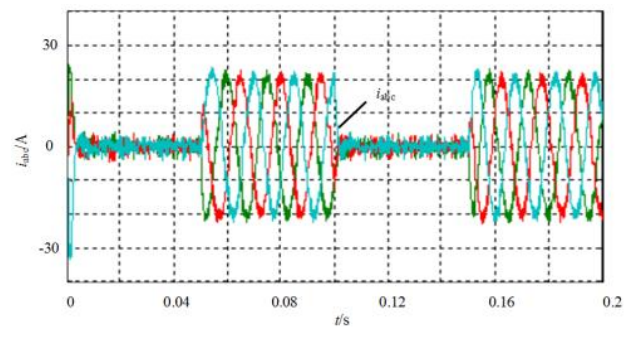

(B) Stator Current on Abc Axle 


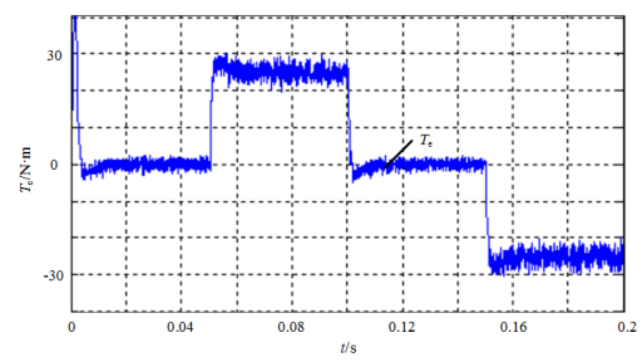

(C) Motor Electromagnetic Torque

\section{Figure 8. Robust Self-Tuning MTPA General Control Features}

The comparison results of MTPA optimization method injected with high frequency. The current vector is operating in the optimal track of MTPA all the time during the whole speed control system, and the current vector of the motor does not exceed the max allowed current loop. In addition, the current track of the dynamic switching procedure of the PMSM speed control system is extremely sparse, thus the dynamic response procedure of the system is extremely rapid, and the whole system is often under stable operation status. The efficiency comparison of the driving system for the air condition compressor under two control methods of MTPA injected with high frequency and $i_{\mathrm{d}}=0$. It can be seen that the MTPA method under rated speed effectively reduces the amplitude of the stator current, and makes the PMSM driving system optimized in aspects of conduction loss, switch loss, DC filter capacitor loss as well as copper loss of the motor. It reduces most significantly the copper loss which is the most intimate with the stator current, and the comprehensive operating efficiency of the system also increases from $91.6 \%$ to $97.4 \%$.

\section{Conclusion}

Aiming at the parameter dependency problem exists in the MTPA control of the traditional PMSM speed regulating system, an optimization method for robust self-tuning efficiency is proposed, the high-frequency power signal processing is made, the high-frequency current controller is designed and the experimental prototype of the speed regulating system for $22 \mathrm{~kW}$ PMSM is built for the method validation in this paper. The following conclusions can be reached: 1) the traditional MTPA method requires to solve an advanced and complex function composed by the inductance $\mathrm{Ld}$ and $\mathrm{Lq}$ as well as the rotor flux $\psi \mathrm{m}$, and it exists a severe parameter dependency problem. 2) The robust MTPA method makes use of the internal relation between the current vector angle and the change rule of the torque $\mathrm{dTe} / \mathrm{dt}$, build the closed-loop control system of the current vector angle $\beta$, and the PMSM speed control system is equipped with strong robustness and high efficiency.

\section{References}

[1] S. Y. Choy, K. M. N. Prasad, T. Y. Wu, M. E. Raghunandan and R. N. Ramanan, "Utilization of plant-based natural coagulants as future alternatives towards sustainable water clarification [J]", Journal of Environmental Sciences, vol. 11, (2014).

[2] J. Wang, S. He and F. Qian, "From Tree Structure to Interactive Network: A Preliminary Study on Transit-Oriented Spatial Development Patterns of Village-Town Communities [J]", China City Planning Review, vol. 04, (2014).

[3] W. W. Yang, Z. M. Guo, L. C. Guo, H. Q. Cao, J. Luo and A. P. Ye, "In situ fabrication and properties of Al N dispersion strengthened 2024 aluminum alloy [J]", International Journal of Minerals Metallurgy and Materials, vol. 12, (2014).

[4] A. R. Khalifeh, A. Dehghan and E. Hajjari, "Dissimilar Joining of AISI 304L/St37 Steels by TIG Welding Process[J]", Acta Metallurgica Sinica (English Letters), vol. 06, (2013).

[5] S. Dorafshan, F. Behnamfar, A. Khamesipour, M. Motosaka, "Condensed hyperelements method of 
non-vertical consistent boundaries for wave propagation analysis in irregular media[J]", Earthquake Engineering and Engineering Vibration, 2013(04)

[6] O. Wallmark, "Modelling of permanent-magnet syn-chronous machines with nonSinusoidal flux linkage", (2002).

[7] S. Najafi and N. C. Kar, "Impact of saturation on transient behavior of interior permanent magnet synchronousmotors", IEEE CCECE/CCGEI, May 7-10, 2006, (2006).

[8] Y. Yan, J. Zhu, H. Lu, Y. Guo and S. Wang, "Study of A PMSM Model Incorporating Structural and Saturation Saliencies", International Conference on Power Electronics and Drives Systems, (2005).

[9] J. Y. Lee, "Determination of perameters considering magnetic nonlinearity in an interior permanent magnet synchronous motor", IEEE Transactions on Magnetics, (2006).

[10] L. Romeral, A. Fabrega, J. Cusido, A. Garcia and J.A. Ortega, "Torque ripple reduction in a PMSM driven by direct torque control", IEEE Annual Power Electronics Specialists Conference, (2008).

[11] Z. Song, J. Li, M. Ouyang, J. Gu, X. Feng and D. Lu, "Rule-based Fault Diagnosis of Hall Sensors and Fault-tolerant Control of PMSM[J]", Chinese Journal of Mechanical Engineering, vol. 04, (2013).

[12] Y. Geng, J. Chen and K. Pahlavan, "Motion detection using RF signals for the first responder in emergency operations: A PHASER project[C]", 2013 IEEE 24nd International Symposium on Personal Indoor and Mobile Radio Communications (PIMRC), London, Britain (2013) September.

[13] S. Li, Y. Geng, J. He and K. Pahlavan, "Analysis of Three-dimensional Maximum Likelihood Algorithm for Capsule Endoscopy Localization”, 2012 5th International Conference on Biomedical Engineering and Informatics (BMEI), Chongqing, China (2012) October, pp. 721-725).

[14] J. Gu, M. Ouyang, J. Li, D. Lu, C. Fang and Y. Ma, "Driving and Braking Control of PM Synchronous Motor Based on Low-resolution Hall Sensor for Battery Electric Vehicle[J]", Chinese Journal of Mechanical Engineering, vol. 01, (2013).

[15] Y. Bian, L. Zhu, H. Lan, A. Li and X. Xu, School of Mechanical Engineering, Tongji University, Shanghai 201804,China, "Regenerative Braking Strategy for Motor Hoist by Ultracapacitor [J]", Chinese Journal of Mechanical Engineering, vol. 02, (2012).

[16] J. Gu, M. Ouyang, J. Li, D. Lu, C. Fang and Y. Ma, "Driving and braking control of PM synchronous motor based on low-resolution hall sensor for battery electric vehicle[J]", Chinese Journal of Mechanical Engineering, vol. 1, (2013).

[17] Z. Lv, T. Yin, Y. Han, Y. Chen and G. Chen, "WebVR--Web Virtual Reality Engine Based on P2P network", Journal of Networks, vol. 6, no. 7, (2011).

[18] W. Li, J. Tordsson and E. Elmroth, "An aspect-oriented approach to consistency-preserving caching and compression of web service response messages", In Web Services (ICWS), 2010 IEEE International Conference on, IEEE, (2010), pp. 526-533.

[19] J. P. Pauwelussen, L Gootjes, C Schröder, K.-U Köhne, S. Jansen and A. Schmeitz, "Full vehicle ABS braking using the SWIFT rigid ring tyre model[J]", Control Engineering Practice, vol. 2, (2003).

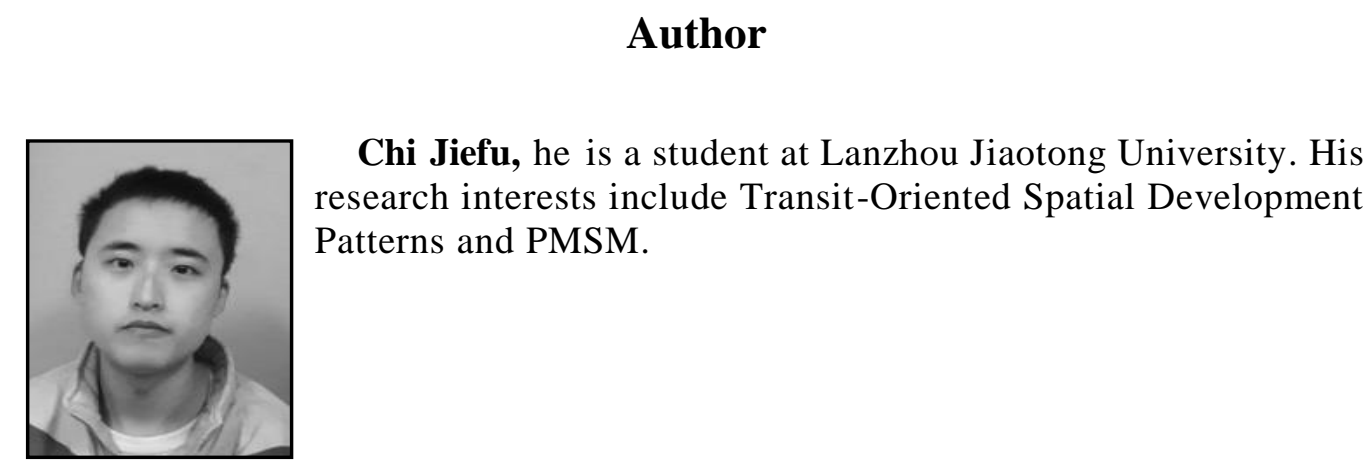


International Journal of Control and Automation

Vol.8, No.11 (2015) 